$10.1016 /$ j.cell.2020.05.027).

They found. the infected mice generated typical interstitial pneumonia and pathology that were similar to those of (COII)-19 patients. Like in humans, the lung is the primary target of this virus, although the eve. heart. and brain could also be infected in accordance with the tissue ACE2 abundance.

They also found that the surviving mice once exposed to a low amount of the virus could better withstand a reinfection at higher amounts. While the naïve-infected mice had a hard time and developed severe symptoms. only mild pneumonia was observed in the mice that have already survived once from viral infection. Which parts of the mouse immune system enable such protection. however. remains unclear.

AII in all. the hACE2 mouse established in this study. partially recapitulating the pathology of (ONID-19 in humans. may provide a valuable platform for testing potential vaccines and therapeutics in the battle with SIRS-COV-2.

\title{
COVID-19
}

\section{Neutralizing Antibody Enters Phase I Clinical Trial}

After the approval by the National Medical Products Administration (NMPA), the first subject from the Shanghai-based Huashan Mospital of Fudan University on the morning of June ? received an injection of JSO 16 - a recombinant, fully human, monoclonal neutralizing antibody against COVID-19. as reported by Xinhua. This is so far the world's first clinical trial concerning using anti-COVID-19 antibody on a healthy human participant following confirming its protective efficacy in nonhuman primates.

The antibody, codeveloped by biopharmaceutical company Junshi Biosciences. the CAs Institute of Microbiology and others. is a reward of treasure-hunting from the blood of a convalescent donor once infected with CONID-19 (Nature 2020, doi: 10.1038/s+1586$020-2 \cdot 381-y)$.

By taking a protein fragment close to the tip of SARS-Cov-2 surface spike protein as a bait. scientists fished out particular memory B-cells that carry the COVID-19 neutralizing antibody on the surface and contain the antibody genes in the core. As a result. they identified two specific human-origin monoclonal antibodies (CA1 and (CB6) that show neutralizing ability in vitro against SARS-Cov-2. Notably. the CB6 antibody can inhibit SARS-CoV-2 infection in rhesus monkeys at both prophylactic and treatment settings. Structural analysis indicates the binding sites of SARS-COV-2 in hACE2, its natural target, are highly overlapped with the binding sites of the virus in the C.B6 antibody. Through joined efforts. this C.OVID-19 neutralizing antibody is currently in phase-I clinical trial. representing a big shot in lighting off the COVID-19 pandemic.

Couple of day earlier, using the same strategy. another group of scientists at the CAS Institute of Microbiology together with others. identified two human-origin monoclonal antibodies that could block the virus binding to its cellular receptor. and hence protect the antibody-given mice from COVII)-19. Turn to page 78 for more detail. 